\title{
Cloud Computing, Networking, and Services
}

\author{
Bhumip Khasnabish • Dijiang Huang • Xiaoying Bai • \\ Paolo Bellavista • Gregorio Martinez • \\ Nick Antonopoulos
}

Received: 14 August 2012/Revised: 31 August 2012/ Accepted: 31 August 2012/

Published online: 9 September 2012

(C) Springer Science+Business Media, LLC 2012

Welcome to the special issue of JNSM on Cloud Computing, Networking, and Services (CCNS).

The concept of Cloud Computing (CC) is based on utilizing distributed (computing) resources for application and services that need massive amount of computing assets for a specific short period of time. The cost of procuring, deploying, and maintaining such massive amount of computing resources for a short time duration is prohibitively high. Therefore, the concept of virtualization has been developed over the last few years to enable sharing of computing resources from distributed clusters of resources (the "Cloud") over the Internet for a fraction of the cost. The early adopters of $\mathrm{CC}$ are small and medium Enterprises. Government

\section{B. Khasnabish}

ZTE USA, Inc., Morristown, NJ, USA

e-mail: vumip1@gmail.com

\section{Huang}

Arizona State University, Phoenix metropolitan area, AZ, USA

e-mail: Dijiang.Huang@asu.edu

X. Bai

Tsinghua University, Beijing, China

e-mail: baixy@tsinghua.edu.cn

P. Bellavista

University of Bologna, Bologna, Italy

e-mail: paolo.bellavista@unibo.it

G. Martinez ( $\square)$

University of Murcia, Murcia, Spain

e-mail: gregorio@um.es

N. Antonopoulos

University of Derby, Derby, UK

e-mail: N.Antonopoulos@derby.ac.uk 
entities and big Corporations are also increasingly adopting private and hybrid (public plus private) Cloud paradigms in order to reduce their capital expenditure (CapEx).

Cloud computing, networking, and their related service (CCNS) management including grid computing (as appropriate) have recently emerged out of hypes to viable computing/networking tools for reducing infrastructure deployment and service management costs without sacrificing the quality of service/experience (QoS/E). There are many definitions of CC; see for example, IETF Website, http://trac.tools. ietf.org/area/app/trac/wiki/Clouds and NIST website, http://www.nist.gov/itl/cloud/. The concept of CC has since been expanded to cover other types of resources as well. These resources include storage resources (DropBox, Microsoft SkyDrive, Google Drive, etc.), communications resources (software defined networking, Networkingas-a-Service or NaaS, etc.), and others. Consequently, the term CC is being commonly used for applications and services that utilize distributed virtualized computing, communications, storage, and any other resources, in general.

Although the virtualization of computing and networking resources, and their self-organizing interconnection is at the heart of it, the methods/mechanisms/tools that are used to expose (visualize) resources and their utilization (the application programming interfaces of APIs) for developing anything $\left(^{*}\right)$ as a service (*aaS) are still ad-hoc and/or proprietary in nature. Security, privacy, and multi-tenancy support requirements add another dimension to the already complex set of CCNS management problems.

The major challenges of adopting cloud computing, networking, and services include seamless cloud service hosting, mobility and service migration, elastic computing using mobile code, distributed CC policy management, and regulations and export control of information.

The general and broad-scope interest raised by the above challenges and related technologies is also demonstrated by the success of submissions to this Special Issue, for which we received more than four dozen papers covering a wide range of topics, including the following:

- Cloud Applications and Services

- API for enabling Public/Private/Hybrid Cloud-based Services

- Virtualization (of any and all resources) and Hosting

- Protocols and Interoperability

- Cloud Service Logging and Monitoring

- Soft and Hard Privacy and Security for Cloud-based Services

- Risk, Resiliency, and SLA (RRS) of Services in Clouds

- Cloud Service and Infrastructure Management

- Reports from CCNS management Experiments and Field Deployments

- Mobility Management in Cloud Computing

However, because of time and space limitations, we are able to accommodate only a limited number of papers, selected after a rigorous review process using relevance, timeliness, and depth of the reported research as criteria. To introduce the interested readers to the contents of this Special Issue, a high-level summary of each selected paper is as follows. 
The paper 'Efficient Online Virtual Network Mapping using Resource Evaluation' by Hao Di, Hongfang Yu, Vishal Anand, Lemin Li, Gang Sun, and Binhong Dong presents an FVN_Sort (First Virtual Node Sorting) function-based On-line Virtual Network Mapping (OVNM) algorithm for maximizing the number of coexisting virtual nodes, and increasing the utilization and revenue obtained from the substrate resources of virtual nodes. By mapping the virtual nodes and links in a coordinated fashion, the authors demonstrate efficient resource utilization without the need of performing excessive mapping.

In the paper, 'Towards Runtime Reconfiguration of Application Control Policies in the Cloud,' Luis M. Vaquero, Daniel Morán, Fermín Galán, and José M. AlcarazCalero describe the use cases and implementation of an adaptive architecture for dynamically controlling the behavior of the applications deployed over the Cloud by using a set of high-level rules. This flexibility allows run-time re-definition of service management policies. Ultimately, this mechanism delivers a successful management of different cloud providers in a highly competitive fashion without sacrificing the associated QoE.

The paper, "A Metric-Based Approach to Assess Risk for "On Cloud" Federated Identity Management' by Patricia Arias-Cabarcos, Florina Almenárez Mendoza, Andrés Marín López, Daniel Díaz Sánchez, and Rosa Sánchez-Guerrero discusses how evidence-based trust management can be utilized for access control and identity management in a dynamic federation environment. The authors also define a set of new metrics using a hierarchical risk aggregation system in these environments.

In the paper, 'Secure and Fast Aggregation of Financial Data in Cloud-Based Expense Tracking Applications,' Juan Camilo Corena and Tomoaki Ohtsuki explain how an architecture based on additive homomorphic cryptosystems and secret sharing schemes can be used to store information securely while still allowing fast aggregation queries at an outsourced untrusted cloud server. The proposal is evaluated in terms of security, server load and complexity of user interaction, computational load at the acquiring terminal, and computational load at the untrusted server.

The paper 'Online Randomization Strategies to Obfuscate User Behavioral Patterns' by Juan E. Tapiador, Julio C. Hernandez-Castro, and Pedro Peris-Lopez examines the vulnerability of a $\mathrm{CC}$ environment. The authors explore how security and privacy leakages derived from the analysis of user activities can be used to capture computationally the working behavioral patterns of the users. This captured information can be used by an adversary for many subversive activities, including (a) predict future activities, (b) detect the occurrence of events of interest, and (c) infer the organization's internal structure. The authors then propose and analyze the concept of disguising user behavior through Online Action Randomization Algorithms (OARA) to randomize user traces in an incremental way.

The final paper 'Efficient Diagnosis Protocol to Enhance the Reliability of a Cloud Computing Environment' by Mao-Lun Chiang discusses the crucial challenge of fault-tolerance in a CC environment. Noting the inefficiency with the traditional Byzantine agreement protocol, the author proposes a FCA (Fast Cloud Agreement) protocol to enhance CC reliability. The FCA can detect a maximum number of faulty processors using a minimum set of messages. 
We sincerely thank the anonymous reviewers who tirelessly contributed to maintain the highest standard of the publication by reviewing the submissions and updated versions of the accepted articles notwithstanding their busy schedules. Our special appreciation also goes to Deep Medhi, for his valuable suggestions during the review process and for constant encouragement to maintain the schedule of publication of this Special Issue. We also thank Paul Brusil for commenting on an earlier draft of this guest editorial.

We enjoyed putting this Special Issue together in this emerging area of CCNS, and fruitfully used this opportunity to have an overview of many emerging and relevant research projects in the area of CCNS during the Special Issue preparation process as well. We hope that the readers will find the selected articles useful and notable in their cloud computing and networking research and implementations.

\section{Author Biographies}

Bhumip Khasnabish, PhD, AMCPM is a Senior Member of IEEE and an emeritus Distinguished Lecturer of the IEEE Communications Society (ComSoc). His current research interests and focus include next-generation networking, platform and services that use cross-domain virtualized computing and communication entities, and tighter cross-layer communications. Bhumip (http://tinyurl.com/bhumip) initiated cloud and data center activities in the IETF, and co-chaired the T\&I activities of ATIS IPTV Interoperability Forum (IIF). He is currently a Senior Director in the Technology Strategy Department of ZTE USA, Inc. Dr. Khasnabish has published (http://tinyurl.com/bhumips-pubs) numerous articles, books, and book chapters, and has been awarded several patents (http://tinyurl.com/bhumips-patents) in his research areas. He is an associate editor of the Journal of Network and Systems Management (JNSM), and edited/co-edited many Special Issues of IEEE Network, IEEE Wireless Communications, IEEE Communications Magazines, and JNSM.

Dijiang Huang received his BS degree from Beijing University of Posts \& Telecommunications, China 1995. He received his MS, and PhD degrees from the University of Missouri-Kansas City, in 2001 and 2004, respectively. He joined Arizona State University (ASU) in 2005 as an assistant professor. He is currently an Associate Professor in the School of Computing Informatics and Decision System Engineering at ASU. His current research interests are computer networking, security, sensor network security, and secure mobile cloud computing. He is currently an associate editor of the Journal of Network and System Management (JNSM) and an editor of the IEEE Communications Surveys and Tutorials. He has served as an organizer for many International conferences and workshops. Dr. Huang's research is supported by NSF, ONR, ARO, Intel, and HP. He is a recipient of ONR Young Investigator Program (YIP) Award 2010 and HP Innovation Research Program (IRP) award 2011 and 2012. He is a senior member of IEEE.

Xiaoying Bai is an Associate Professor at the Department of Computer Science and Technology of Tsinghua University, Beijing, China. She received her PhD degree in computer science in 2001 from Arizona State University in the USA. Her major research area is software engineering, especially modeldriven testing and test automation techniques in various software paradigms such as distributed computing, service oriented architecture, cloud computing and embedded systems. She has published over 90 papers in journals and conferences, and is the co-author of a Chinese book on "Service-Oriented Software Engineering". She is an associate editor of the International Journal on Software Engineering and Knowledge Engineering (IJSEKE), an editor of Service Oriented Computing and Application (SOCA), and guest editor of several special issues of IJSEKE, SOCA, Journal of Systems and Software, and JNSM. Her work has been supported by National Science Foundation China, National High Technology Program 863, National Basic Research Program of China, and industry companies like IBM, Freescale, and Fujitsu. 
Paolo Bellavista is an Associate Professor of computer engineering at the University of Bologna, where he has received his Laurea degree and $\mathrm{PhD}$. He is a senior member of the IEEE and of the ACM, and serves on the Editorial Boards of the IEEE Communications Magazine, the IEEE Transactions on Computers, the IEEE Transactions on Services Computing, the IEEE Transactions on Network and Service Management, the Springer Journal of Network and Systems Management, and the Elsevier Pervasive and Mobile Computing Journal. His research activities span from mobile computing to mobile agent-based middleware, from pervasive wireless computing to location/context-aware services, from vehicular/sensor wireless ad hoc networks to adaptive multimedia. He is co-editor of the "Handbook of Mobile Middleware" and authored more than 140 journal/conference papers. Additional information at: http://lia.deis.unibo.it/Staff/PaoloBellavista/.

Gregorio Martinez is an Associate Professor in the Department of Information and Communications Engineering of the University of Murcia. His research interests include security and management of distributed communication networks. He received the MSc and $\mathrm{PhD}$ degrees in Computer Science from the University of Murcia. He has published more than a hundred journal articles and conference papers. He has been involved as collaborator or supervisor in several open-source software projects. He is also on the editorial or review board of more than 20 international journals. Additional information at: http://webs.um.es/gregorio/.

Nick Antonopoulos is currently the Head of the School of Computing \& Mathematics and Assistant Dean (Research) of the Faculty of Business, Computing \& Law at the University of Derby. Prior to joining the University of Derby in 2009 he was a Senior Lecturer (US Associate Professor) and Director of the MSc Degrees at the Department of Computing, University of Surrey, UK. He holds a BSc in Physics (1st class) from the University of Athens in 1993, an MSc in Information Technology from Aston University in 1994 and a PhD in Computer Science from the University of Surrey in 2000. He has over 14 years of academic experience during which he has published over 110 articles in fully refereed journals and international conferences. He has received a number of best paper awards in conferences and graduated $7 \mathrm{PhD}$ students. He has edited two books in the field of P2P, Grids and Cloud Computing published by IGI Global and Springer respectively. He is on the editorial board of the Springer journal of Peer-to-Peer Networking and Applications and co-editor-in-chief of the Springer journal of Cloud Computing. He is a Fellow of the UK Higher Education Academy and a Fellow of the British Computer Society. 